\title{
ON MODULAR FIBONACCI AND TRIBONACCI TABLES
}

\author{
EunMi ChOI*
}

\begin{abstract}
The work is devoted to study Fibonacci and tribonacci numbers. We study the modular formulas and the periods of the sequences.
\end{abstract}

\section{Introduction}

The investigation of Fibonacci sequence $F_{n}=F_{n-1}+F_{n-2}$ with $F_{0}=$ $0, F_{1}=1$ has been extended to algebraic aspect since D.D. Wall [7] in 1960. In particular researches including [1], [3], [6] were devoted to study Fibonacci sequences by modulo $n$ in connection with order and period. The Fibonacci sequence has been studied in some arithmetic triangle forms, for instance all Fibonacci numbers appear along the diagonal of the Pascal triangle. Instead of triangle, if we display the Fibonacci sequence in rectangle form [2], say a rectangle with three columns, and if we take each numbers by mod $F_{3}=2$ then we have the following tables

$\begin{array}{llll}1 & 1 & 2 \\ 3 & 5 & 8 \\ 13 & 21 & 34 \\ 55 & 89 & \cdots\end{array} \quad$ and $\quad \begin{array}{lll}1 & 1 & 0 \\ 1 & 1 & 0 \\ 1 & 1 & 0 \\ 1 & 1 & \ldots\end{array}$

We call the left table the 3 columns Fibonacci table. It shows $(2 \cdot 2) 34+$ $8=144$ and $(2 \cdot 2) 55+13=233$, where these can be expressed by

$$
2 F_{3} F_{9}+F_{6}=F_{12} \text { and } 2 F_{3} F_{10}+F_{7}=F_{13} .
$$

And the right table, called the 3 columns modular table, shows a repetition of modular Fibonacci numbers. Similarly the 4 columns Fibonacci and its modular table by $\bmod T_{4}=3$

Received April 25, 2013; Accepted July 18, 2013.

2010 Mathematics Subject Classification: Primary 11B37, 15A36, 11P.

Key words and phrases: Fibonacci-Lucas-tribonacci sequences.

* This work was supported by Hannam University Research Fund 2013. 


$\begin{array}{llllllll}1 & 1 & 2 & 3 & 1 & 1 & 2 & 0 \\ 5 & 8 & 13 & 21 & 2 & 2 & 1 & 0 \\ 34 & 55 & 89 & 144 & 1 & 1 & 2 & 0 \\ 233 & 377 & 610 & \ldots & 2 & 2 & 1 & \ldots\end{array}$

show that $(2(3)+1) 233-34=1597$, i.e., $\left(2 F_{4}+F_{1}\right) F_{13}-F_{9}=F_{17}$. Thus for instance, the 25th Fibonacci number $F_{25}$ can be obtained by

$$
\left(2 F_{4}+F_{1}\right) F_{21}+(-1)^{3} F_{17}=(7) 10946-1597=75025=F_{25} .
$$

When we say tribonacci sequence $T_{n}$, we mean a sequence like $F_{n}$, but instead of two initial 0 and 1 , the tribonacci sequence starts with three values 0,0 and 1 and each term afterwards is the sum of the preceding three terms. Hence $T_{n}=T_{n-1}+T_{n-2}+T_{n-3}$ with $T_{0}=0, T_{1}=T_{2}=1$, and the first a few tribonacci numbers are $\{0,0,1,1,2,4,7,13,24,44, \cdots\}$.

In this work we study Fibonacci and tribonacci sequence by displaying in rectangle form. By taking modular, we will find periods of the sequences.

\section{Fibonacci table and modular Fibonacci table}

The Fibonacci number $F_{n}$ can be extended to negative $n$ such that $F_{-1}=1, F_{-2}=-1$ and $F_{-3}=2$, and $F_{-n}=(-1)^{n+1} F_{n}$ for all $n \in \mathbb{Z}$.

Lemma 2.1. Let $n, t \in \mathbb{Z}$.

(1) $F_{n+3}=2 F_{3} F_{n}+F_{n-3}$. If $n=3 t+r(1 \leq r \leq 3)$ then $F_{n}=$ $2 F_{3} F_{3(t-1)+r}+F_{3(t-2)+r}$. So $F_{3 t+r} \equiv F_{3(t-2)+r}\left(\bmod F_{3}\right)$.

(2) $F_{n+4}=\left(2 F_{4}+F_{1}\right) F_{n}-F_{n-4}$.

(3) If $n=4 t+r(1 \leq r \leq 4)$ then $F_{n}=\left(2 F_{4}+F_{1}\right) F_{4(t-1)+r}-F_{4(t-2)+r}$. So, $F_{4 t+r} \equiv F_{4(t-1)+r}-F_{4(t-2)+r}\left(\bmod F_{4}\right)$.

Proof. We have seen that $F_{n+3}=2 F_{3} F_{n}+F_{n-3}$ for $n=1,2$. Assume $F_{i+3}=2 F_{3} F_{i}+F_{i-3}$ for all $i \leq n$. Then (1) is clear that

$$
\begin{aligned}
F_{(n+1)+3} & =F_{n+3}+F_{(n-1)+3} \\
& =2 F_{3} F_{n}+F_{n-3}+2 F_{3} F_{n-1}+F_{n-4} \\
& =2 F_{3}\left(F_{n}+F_{n-1}\right)+F_{n-3}+F_{n-4}=2 F_{3} F_{n+1}+F_{(n+1)-3} .
\end{aligned}
$$

The rest can be proved similarly.

This can be generalized as follows. 
THEOREM 2.2. If $n \in \mathbb{Z}$ then $F_{n+k}=\left(2 F_{k}+F_{k-3}\right) F_{n}+(-1)^{k-1} F_{n-k}$ for all $k \geq 3$. If we write $n=k t+r(t, r \in \mathbb{Z}, 1 \leq r \leq k)$ then

$$
F_{n}=F_{k t+r}=\left(2 F_{k}+F_{k-3}\right) F_{k(t-1)+r}+(-1)^{k-1} F_{k(t-2)+r}
$$

and $F_{k t+r} \equiv F_{k-3} F_{k(t-1)+r}+(-1)^{k-1} F_{k(t-2)+r}\left(\bmod F_{k}\right)$.

Proof. The cases of $k=3$ or 4 are due to Lemma 2.1. We now will consider the 5 columns Fibonacci table:

$\begin{array}{lllll}1 & 1 & 2 & 3 & 5 \\ 8 & 13 & 21 & 34 & 55 \\ 89 & 144 & 233 & 377 & 610 \\ 987 & 1597 & 2584 & 4181 & \cdots\end{array}$

It shows $(2(5)+1)(377)+34=4181$, i.e., $\left(2 F_{5}+F_{2}\right) F_{16}+F_{11}=F_{21}$. So

$$
F_{n+5}=\left(2 F_{5}+F_{2}\right) F_{5}+F_{n-5},
$$

thus

$$
F_{n+i}=\left(2 F_{i}+F_{i-3}\right) F_{n}+(-1)^{i-1} F_{n-i} \text { for } 3 \leq i \leq 5 .
$$

Assume it is true for $i \leq k-1$. Then in the $k$ columns Fibonacci table,

$$
\begin{aligned}
& F_{n+k} \\
&= F_{n+(k-1)}+F_{n+(k-2)} \\
&=\left(2 F_{k-1}+F_{k-4}\right) F_{n}+(-1)^{k-2} F_{n-(k-1)}+\left(2 F_{k-2}+F_{k-5}\right) F_{n}+ \\
&(-1)^{k-1} F_{n-(k-2)} \\
&=\left(2\left(F_{k-1}+F_{k-2}\right)+F_{k-4}+F_{k-5}\right) F_{n}+(-1)^{k-3}\left(-F_{n-(k-1)}+F_{n-(k-2)}\right) \\
&=\left(2 F_{k}+F_{k-3}\right) F_{n}+(-1)^{k-1} F_{n-k},
\end{aligned}
$$

since $F_{n-k}+F_{n-(k-1)}=F_{n-(k-2)}$. Moreover for $n=k t+r(1 \leq r \leq k)$,

$$
\begin{aligned}
F_{k t+r}=F_{(n-k)+k} & =\left(2 F_{k}+F_{k-3}\right) F_{n-k}+(-1)^{k-1} F_{n-2 k} \\
& =\left(2 F_{k}+F_{k-3}\right) F_{k(t-1)+r}+(-1)^{k-1} F_{k(t-2)+r} .
\end{aligned}
$$

Thus $F_{k t+r} \equiv F_{k-3} F_{k(t-1)+r}+(-1)^{k-1} F_{k(t-2)+r}\left(\bmod F_{k}\right)$.

It shows that $F_{k t+r}$ is a combination of $F_{k(t-1)+r}$ and $F_{k(t-2)+r}$ with coefficient $2 F_{k}+F_{k-3}$ and $(-1)^{k-1}$. Inductively we have the following.

Theorem 2.3. Let $n=k t+r(1 \leq r \leq k)$. Then every $F_{n}$ can be written by only four Fibonacci numbers $F_{k}, F_{k-3}, F_{r}$ and $F_{k+r}$. Moreover if $F_{k t+r}=\theta_{1} F_{k+r}+\theta_{2} F_{r}$ with $\theta_{1}, \theta_{2} \in \mathbb{Z}$ then $F_{k(t+1)+r}=$ $\left(\left(2 F_{k}+F_{k-3}\right) \theta_{1}+\theta_{2}\right) F_{k+r}+\theta_{1} F_{r}$. 
Proof. Theorem 2.2 implies that $F_{k t+r}=\mu F_{k(t-1)+r}+(-1)^{k-1} F_{k(t-2)+r}$ with $\mu=2 F_{k}+F_{k-3}$. We first assume $k$ is odd. Then

$$
\begin{aligned}
& F_{2 k+r}=\mu F_{k+r}+F_{r} \\
& F_{3 k+r}=\mu F_{2 k+r}+F_{k+r}=\mu\left(\mu F_{k+r}+F_{r}\right)+F_{k+r}=\left(\mu^{2}+1\right) F_{k+r}+\mu F_{r} \\
& F_{4 k+r}=\mu F_{3 k+r}+F_{2 k+r}=\left(\mu^{3}+2 \mu\right) F_{k+r}+\left(\mu^{2}+1\right) \mu F_{r} \\
& F_{5 k+r}=\mu F_{4 k+r}+F_{3 k+r}=\left(\mu^{4}+3 \mu^{2}+1\right) F_{k+r}+\left(\mu^{3}+2 \mu\right) \mu F_{r} .
\end{aligned}
$$

The first coefficient in this stage is $\mu$ times the first coefficient in previous step added to the second coefficient in previous step, while the second coefficient in this stage is the first coefficient in the previous step.

Now suppose that this pattern is true for all $j$ th stages $(1 \leq j<t)$. That is, we assume that if $F_{k(j-1)+r}=\chi_{1} F_{k+r}+\chi_{2} F_{r}$ then $F_{k j+r}=$ $\theta_{1} F_{k+r}+\theta_{2} F_{r}$ where $\theta_{1}=\mu \chi_{1}+\chi_{2}$ and $\theta_{2}=\chi_{1}$ for $\chi_{1}, \chi_{2} \in \mathbb{Z}$. Due to Theorem 2.2,

$$
\begin{aligned}
F_{k(j+1)+r} & =\mu F_{k j+r}+F_{k(j-1)+r} \\
& =\mu\left(\theta_{1} F_{k+r}+\theta_{2} F_{r}\right)+\chi_{1} F_{k+r}+\chi_{2} F_{r} \\
& =\left(\mu \theta_{1}+\chi_{1}\right) F_{k+r}+\left(\mu \theta_{2}+\chi_{2}\right) F_{r} \\
& =\left(\mu \theta_{1}+\theta_{2}\right) F_{k+r}+\left(\mu \chi_{1}+\chi_{2}\right) F_{r}=\left(\mu \theta_{1}+\theta_{2}\right) F_{k+r}+\theta_{1} F_{r} .
\end{aligned}
$$

The case when $k$ is even can be prove similarly.

It gives a good way to compute $F_{n}$ by knowing only a few information about $F_{k}, F_{k-3}, F_{r}$ and $F_{k+r}$. The first three are in the first row while the last one is in the second row of the $k$ columns Fibonacci table.

EXAMPLE 2.4. For 50th Fibonacci $F_{50}$, take $k=7$ for instance, then

$$
\begin{aligned}
F_{50} & =F_{7 \cdot 7+1}=\mu F_{7 \cdot 6+1}+F_{7 \cdot 5+1}=\left(\mu^{2}+1\right) F_{7 \cdot 5+1}+\mu F_{7 \cdot 4+1} \\
& =\left(\mu\left(\mu^{2}+1\right)+\mu\right) F_{7 \cdot 4+1}+\left(\mu^{2}+1\right) F_{7 \cdot 3+1} \\
& =\left(\mu\left(\mu^{3}+2 \mu\right)+\mu^{2}+1\right) F_{7 \cdot 3+1}+\left(\mu^{3}+2 \mu\right) F_{7 \cdot 2+1} \\
& =\left(\mu\left(\mu^{4}+3 \mu^{2}+1\right)+\mu^{3}+2 \mu F_{7 \cdot 2+1}+\left(\mu^{4}+3 \mu^{2}+1\right) F_{7+1}\right. \\
& =\left(\mu\left(\mu^{5}+4 \mu^{3}+3 \mu\right)+\mu^{4}+3 \mu^{2}+1\right) F_{7+1}+\left(\mu^{5}+4 \mu^{3}+3 \mu\right) F_{1} \\
& =12,586,269,025
\end{aligned}
$$

by plugging $F_{7}=13, F_{4}=3, F_{1}=1, F_{8}=21$ and $\mu=2 F_{7}+F_{4}=29$.

Corollary 2.5 .

(1) Every $F_{k t} \equiv 0\left(\bmod F_{k}\right)$. If $n \mid m$ then $F_{n} \mid F_{m}$ for every $n, m \in \mathbb{Z}$.

(2) If $k$ is even, every $(t)$ th row is congruent to $(t \pm 2)$ th row by mod $F_{k}$ in the $k$ columns modular table. The first two rows are repeated in order, so the modular Fibonacci sequence by $\bmod F_{k}$ is periodic of length $2 k$. 
(3) If $k$ is odd, every ( $t)$ th row is congruent to $(t \pm 2)$ th row with negative sign by mod $F_{k}$ in the $k$ columns modular table. The first four rows are repeated in order, so the modular Fibonacci sequence by mod $F_{k}$ is periodic of length $4 k$.

Proof. Since $F_{k t+r}$ is written by $F_{k+r}$ and $F_{r}, F_{k t}=F_{k(t-1)+k}$ is a linear combination of $F_{k+k}$ and $F_{k}$, and again by $F_{k}$ and $F_{0}$. But since both $F_{k}$ and $F_{0}$ are 0 by $\bmod F_{k}$, it follows $F_{k t} \equiv 0\left(\bmod F_{k}\right)$. The rest are due to Theorem 2.3.

Note that $F_{k} \mid F_{k t}$ in (1) has been proved by various ways. One way is due to show $\operatorname{per}_{F}(n)=l c m\left(\operatorname{per}_{F}\left(p_{1}\right), \cdots, \operatorname{per}_{F}\left(p_{s}\right)\right)$ for all primes $p_{i} \mid n$ [7]. The other method is to use the fact $\operatorname{gcd}\left(F_{k}, F_{t}\right)=F_{\operatorname{gcd}(k, t)}$ in [4]. Of course $F_{k} \mid F_{k t}$ can be proved by induction on $t$ after fixing $k$. However it seems that the proof using the $k$ columns modulo table is more convenient than any other methods. Owing to Corollary 2.4, we can construct the modular Fibonacci tables for $5 \leq k \leq 8$ :

\begin{tabular}{rrrrlll|rrrrrrrrr}
\hline \multicolumn{1}{c|}{$\bmod \left(F_{5}=5\right)$} & & & & $\bmod \left(F_{6}=8\right)$ \\
\hline \hline 1 & 1 & 2 & 3 & 0 & & & 1 & 1 & 2 & 3 & 5 & 0 & & \\
3 & 3 & 1 & 4 & 0 & & & 5 & 5 & 2 & 7 & 1 & 0 & & \\
-1 & -1 & -2 & -3 & 0 & & & 1 & 1 & 2 & 3 & 5 & 0 & & \\
-3 & -3 & -1 & -4 & $\cdots$ & & & 5 & 5 & 2 & 7 & 1 & $\cdots$ & & \\
\hline \multicolumn{2}{c}{$\bmod \left(F_{7}=13\right)$} & & & & & \multicolumn{1}{|c|}{$\bmod \left(F_{8}=21\right)$} & & & & \\
\hline \hline & 1 & 2 & 3 & 5 & 8 & 0 & 1 & 1 & 2 & 3 & 5 & 8 & 13 & 0 \\
8 & -5 & 3 & -2 & 1 & -1 & 0 & 13 & 13 & 5 & 18 & 2 & 20 & 1 & 0 \\
-1 & -1 & -2 & -3 & -5 & -8 & 0 & 1 & 1 & 2 & 3 & 5 & 8 & 13 & 0 \\
-8 & 5 & -3 & 2 & -1 & 1 & $\cdots$ & 13 & 13 & 5 & 18 & 2 & 20 & 1 & $\cdots$ \\
\hline
\end{tabular}

\section{Tribonacci table and modular tribonacci table}

In this section we deal with tribonacci sequence $T_{n}=T_{n-1}+T_{n-2}+$ $T_{n-3}$ with $T_{0}=0$ and $T_{1}=T_{2}=1$. Similar to Fibonacci numbers, $T_{n}$ can be extended to negative $n$ such that $T_{-1}=0, T_{-2}=1, T_{-3}=-1$ and $T_{-4}=0$, etc. Let us consider the 4 columns tribonacci table

$\begin{array}{llll}1 & 1 & 2 & 4 \\ 7 & 13 & 24 & 44 \\ 81 & 149 & 274 & 504 \\ 927 & 1705 & 3136 & \ldots\end{array}$


It is clear to see that

$T_{16}=(11) 504+(5) 44+4=\left(3 T_{4}-1\right) T_{12}+\left(T_{4}+1\right) T_{8}+T_{4}=5768$

$T_{19}=(11) 3136+(5) 274+24=\left(3 T_{4}-1\right) T_{15}+\left(T_{4}+1\right) T_{11}+T_{7}=35890$

Theorem 3.1. Let $n=k t+r(1 \leq r \leq k)$. Then for $4 \leq k \leq 6$,

$$
T_{k t+r}=\mu_{1} T_{k(t-1)+r}+\mu_{2} T_{k(t-2)+r}+T_{k(t-3)+r},
$$

that is, $T_{n}=\mu_{1} T_{n-k}+\mu_{2} T_{n-2 k}+\mu_{3} T_{n-3 k}$, where the coefficients $\left(\mu_{1}, \mu_{2}, \mu_{3}\right)$ depending on $k$ are as follows

\begin{tabular}{l||l|l|l}
\hline & $k=4$ & $k=5$ & $k=6$ \\
\hline$\left(\mu_{1}, \mu_{2}, \mu_{3}\right)$ & $\left(3 T_{4}-1, T_{4}+1,1\right)$ & $\left(3 T_{5}, 1,1\right)$ & $\left(3 T_{6},-T_{6}+2,1\right)$ \\
\hline
\end{tabular}

Proof. When $k=4$ we will prove

$$
T_{n}=\left(3 T_{4}-1\right) T_{n-4}+\left(T_{4}+1\right) T_{n-8}+T_{n-12} .
$$

If $n=12$ then $\left(3 T_{4}-1\right) T_{8}+\left(T_{4}+1\right) T_{4}+T_{0}=504=T_{12}$. Assume that

$$
T_{i}=\mu_{1} T_{i-4}+\mu_{2} T_{i-8}+T_{i-12} \text { for all } 12 \leq i \leq n
$$

with $\mu_{1}=3 T_{4}-1$ and $\mu_{2}=T_{4}+1$. Then

$$
\begin{aligned}
& \mu_{1} T_{(n+1)-4}+\mu_{2} T_{(n+1)-8}+T_{(n+1)-12} \\
= & \mu_{1}\left(T_{n-4}+T_{(n-4)-1}+T_{(n-4)-2}\right)+\mu_{2}\left(T_{n-8}+T_{(n-8)-1}+T_{(n-8)-2}\right) \\
& \quad+\left(T_{n-12}+T_{(n-12)-1}+T_{(n-12)-2}\right) \\
= & \left(\mu_{1} T_{n-4}+\mu_{2} T_{n-8}+T_{n-12}\right)+\left(\mu_{1} T_{(n-1)-4}+\mu_{2} T_{(n-1)-8}+T_{(n-1)-12}\right) \\
& \quad+\left(\mu_{1} T_{(n-2)-4}+\mu_{2} T_{(n-2)-8}+T_{(n-2)-12}\right) \\
= & T_{n}+T_{n-1}+T_{n-2}=T_{n+1} .
\end{aligned}
$$

If $n<12$ then by considering negative tribonaccis $T_{-1}=0, T_{-2}=1$, etc., without loss of generality we have

$T_{n}=\left(3 T_{4}-1\right) T_{n-4}+\left(T_{4}+1\right) T_{n-8}+T_{n-12}$ for all $n$.

Similarly from the 5 columns tribonacci table

$\begin{array}{lllll}1 & 1 & 2 & 4 & 7 \\ 13 & 24 & 44 & 81 & 149 \\ 274 & 504 & 927 & 1705 & 3136 \\ 5768 & 10609 & 19513 & 35890 & \ldots\end{array}$

we can find that

$$
\left\{\begin{array}{l}
T_{17}=10690=(21) 504+24+1=\left(3 T_{5}\right) T_{12}+T_{7}+T_{2} \\
T_{23}=410744=(21) 19513+927+44=\left(3 T_{5}\right) T_{18}+T_{13}+T_{8}
\end{array}\right.
$$

Moreover from the 6 columns tribonacci table

$\begin{array}{llllll}1 & 1 & 2 & 4 & 7 & 13 \\ 24 & 44 & 81 & 149 & 274 & 504 \\ 927 & 1705 & 3136 & 5768 & 10609 & 19513 \\ 35890 & 66012 & 121415 & 223317 & 410744 & \cdots\end{array}$


it can be seen that

$\left\{\begin{array}{l}T_{20}=66012=(39) 1705-(13-2) 44+1=\left(3 T_{6}\right) T_{14}-\left(T_{6}-2\right) T_{8}+T_{2} \\ T_{22}=223317=(39) 5768-(13-2) 149+4=\left(3 T_{6}\right) T_{16}-\left(T_{6}-2\right) T_{10}+T_{4}\end{array}\right.$

Now we assume that, for $k=5$ or 6 the equality

$$
T_{n+k i}=\mu_{1} T_{n+k(i-1)}+\mu_{2} T_{n+k(i-2)}+\mu_{3} T_{n+k(i-3)}
$$

with $\left(\mu_{1}, \mu_{2}, \mu_{3}\right)=\left(3 T_{5}, 1,1\right)$ or $\left(3 T_{6},-T_{6}+2,1\right)$ hold for all $1 \leq i<v$. Then

$$
\begin{aligned}
T_{n+k v} & =T_{(n+k)+k(v-1)} \\
& =\mu_{1} T_{(n+k)+k(v-2)}+\mu_{2} T_{(n+k)+k(v-3)}+\mu_{3} T_{(n+k)+k(v-4)} \\
& =\mu_{1} T_{n+k(v-1)}+\mu_{2} T_{n+k(v-2)}+\mu_{3} T_{n+k(v-3)},
\end{aligned}
$$

it proves the theorem.

Theorem 3.2. Let $n=k t+r(1 \leq r \leq k)$. Then for $7 \leq k \leq 10$,

$$
T_{k t+r}=\mu_{1} T_{k(t-1)+r}+\mu_{2} T_{k(t-2)+r}+\mu_{3} T_{k(t-3)+r}
$$

where the coefficients $\left(\mu_{1}, \mu_{2}, \mu_{3}\right)$ are determined as follows.

\begin{tabular}{l|l|l|l}
\hline$k=7$ & 8 & 9 & 10 \\
\hline$\left(3 T_{7}-1,15,1\right)$ & $\left(3 T_{8}-1,-1,1\right)$ & $\left(3 T_{9}-2,-23,1\right)$ & $\left(3 T_{10}-4,41,1\right)$ \\
\hline
\end{tabular}

Proof. The 7 columns tribonacci tables

$\begin{array}{lllllll}1 & 1 & 2 & 4 & 7 & 13 & 24 \\ 44 & 81 & 149 & 274 & 504 & 927 & 1705 \\ 3136 & 5768 & 10609 & 19513 & 35890 & 66012 & 121415 \\ 223317 & 410744 & 755476 & 1389537 & 2555757 & 4700770 & \cdots\end{array}$

shows that

$\left\{\begin{array}{l}T_{22}=223317=(71) 3136+(15) 44+1=\left(3 T_{7}-1\right) T_{15}+15 T_{8}+T_{1} \\ T_{27}=4700770=(71) 66012+(15) 927+13=\left(3 T_{7}-1\right) T_{20}+15 T_{13}+T_{6} .\end{array}\right.$

Thus similar to the proof of Theorem 3.1, it can be proved

$$
T_{7 t+r}=\left(3 T_{7}-1\right) T_{7(t-1)+r}+15 T_{7(t-2)+r}+T_{7(t-3)+r}(1 \leq r \leq 7) .
$$

From the 8 columns tribonacci table

$\begin{array}{llllllll}1 & 1 & 2 & 4 & 7 & 13 & 24 & 44 \\ 81 & 149 & 274 & 504 & 927 & 1705 & 3136 & 5768 \\ 10609 & 19513 & 35890 & 66012 & 121415 & 223317 & \cdots & \\ 1389537 & 2555757 & 4700770 & 8646064 & 15902591 & \ldots & & \end{array}$

we find that

$$
\left\{\begin{array}{l}
T_{25}=1389537=(131) 10609-(3) 81+=\left(3 T_{8}-1\right) T_{17}-3 T_{9}+T_{1} \\
T_{29}=15902591=(131) 121415-(3) 927+7=\left(3 T_{8}-1\right) T_{21}-3 T_{13}+T_{5}
\end{array}\right.
$$


hence $T_{8 t+r}=\left(3 T_{8}-1\right) T_{8(t-1)+r}-T_{8(t-2)+r}+T_{8(t-3)+r}(1 \leq r \leq 8)$.

The 9 and 10 columns tribonacci tables show that, for instance

$$
\left\{\begin{array}{l}
T_{28}=(241) 35890-(23) 149+1=\left(3 T_{9}-2\right) T_{19}-(23) T_{10}+T_{1} \\
T_{33}=(241) 755476-(23) 3136+13=\left(3 T_{9}-2\right) T_{24}-(23) T_{15}+T_{6}
\end{array}\right.
$$

hence $T_{9 t+r}=\left(3 T_{9}-2\right) T_{9(t-1)+r}-23 T_{9(t-2)+r}+T_{9(t-3)+r}(1 \leq r \leq 9)$. And

$$
\left\{\begin{array}{l}
T_{31}=(443) 121415+(41) 274+1=\left(3 T_{10}-4\right) T_{21}+(41) T_{11}+T_{1} \\
T_{34}=(443) 410744+(41) 927+2=\left(3 T_{10}-4\right) T_{24}+(41) T_{14}+T_{4}
\end{array}\right.
$$

so $T_{10 t+r}=\left(3 T_{10}-4\right) T_{10(t-1)+r}+41 T_{10(t-2)+r}+T_{10(t-3)+r}(1 \leq r \leq 10)$.

Analogue to the proof of Theorem 3.1, the induction yields the identity $T_{k t+r}=\mu_{1} T_{k(t-1)+r}+\mu_{2} T_{k(t-2)+r}+\mu_{3} T_{k(t-3)+r}$.

We note that Theorem 3.1 and 3.2 can be extended to negative $n$ of $T_{n}$ by taking $T_{-1}=0, T_{-2}=1, T_{-3}=-1, \cdots$. The following theorem provides an efficient method for $T_{n}$ with $n<0$.

Theorem 3.3. Let $-n=k(-t)+r<0(1 \leq r \leq k, t>0)$. Then

$$
T_{-n}=T_{k(-t)+r}=-\mu_{2} T_{k(-t+1)+r}-\mu_{1} T_{k(-t+2)+r}+T_{k(-t+3)+r}
$$

for $4 \leq k \leq 10$, where the coefficients $\mu_{1}$ and $\mu_{2}$ (depending on $k$ ) are as in Theorem 3.1 and 3.2.

Proof. Due to Theorem 3.1 and 3.2,

$$
\mu_{1} T_{k(-t+2)+r}+\mu_{2} T_{k(-t+1)+r}+\mu_{3} T_{k(-t)+r}=T_{k(-t+3)+r} .
$$

Since $\mu_{3}=1$ for all $4 \leq k \leq 10$,

$$
T_{k(-t)+r}=-\mu_{1} T_{k(-t+2)+r}-\mu_{2} T_{k(-t+1)+r}+T_{k(-t+3)+r} .
$$

For instance, $T_{-16}==-T_{5(-3)+4}-21 T_{5(-2)+4}+T_{5(-1)+4}=56$.

Theorem 3.4. $T_{k t+r}(4 \leq k \leq 10)$ is a linear combination of $T_{2 k+r}$, $T_{k+r}$ and $T_{r}$. 
Proof. Due to Theorem 3.1 and 3.2, we have

$$
\begin{aligned}
T_{k t+r} \\
=\mu_{1} T_{k(t-1)+r}+\mu_{2} T_{k(t-2)+r}+\mu_{3} T_{k(t-3)+r} \\
=\mu_{1}\left(\mu_{1} T_{k(t-2)+r}+\mu_{2} T_{k(t-3)+r}+\mu_{3} T_{k(t-4)+r}\right)+\mu_{2} T_{k(t-2)+r} \\
\quad+\mu_{3} T_{k(t-3)+r} \\
=\left(\mu_{1}^{2}+\mu_{2}\right) T_{k(t-2)+r}+\left(\mu_{1} \mu_{2}+\mu_{3}\right) T_{k(t-3)+r}+\mu_{1} T_{k(t-4)+r} \\
=\left(\mu_{1}^{2}+\mu_{2}\right)\left(\mu_{1} T_{k(t-3)+r}+\mu_{2} T_{k(t-4)+r}+\mu_{3} T_{k(t-5)+r}\right) \\
\quad+\left(\mu_{1} \mu_{2}+\mu_{3}\right) T_{k(t-3)+r}+\mu_{1} T_{k(t-4)+r} \\
=\left(\mu_{1}\left(\mu_{1}^{2}+\mu_{2}\right)+\left(\mu_{1} \mu_{2}+\mu_{3}\right)\right) T_{k(t-3)+r}+\left(\mu_{2}\left(\mu_{1}^{2}+\mu_{2}\right)+\mu_{3}\right) T_{k(t-4)+r} \\
\quad+\left(\mu_{1}^{2}+\mu_{2}\right) \mu_{3} T_{k(t-5)+r}
\end{aligned}
$$

Hence after some steps, if we write

$$
T_{k t+r}=\theta_{1} T_{k(t-i-1)+r}+\theta_{2} T_{k(t-i-2)+r}+\theta_{3} T_{k(t-i-3)+r}
$$

for some $i \in \mathbb{Z}$, then the next stage should be

$T_{k t+r}=\left(\mu_{1} \theta_{1}+\theta_{2}\right) T_{k(t-i-2)+r}+\left(\mu_{2} \theta_{1}+\theta_{3}\right) T_{k(t-i-3)+r}+\mu_{3} \theta_{1} T_{k(t-i-4)+r}$. Thus if $i=t-4$ then $T_{k t+r}$ is a combination of $T_{2 k+r}, T_{k+r}$ and $T_{r}$.

EXAmPle 3.5. For $T_{50}$, take $k=7$ for instance, then

$$
T_{50}=T_{7(7)+1}=\mu_{1} T_{7(6)+1}+\mu_{2} T_{7(5)+1}+\mu_{3} T_{7(4)+1}
$$

with $\left(\mu_{1}, \mu_{2}, \mu_{3}\right)=\left(3 T_{7}-1,15,1\right)=(71,15,1)$. So we have

$$
\begin{aligned}
& T_{50} \\
& =71 T_{7(6)+1}+15 T_{7(5)+1}+T_{7(4)+1} \\
& =(71 \cdot 71+15) T_{7(5)+1}+(15 \cdot 71+1) T_{7(4)+1}+71 T_{7(3)+1} \\
& =5056 T_{7(5)+1}+1066 T_{7(4)+1}+71 T_{7(3)+1} \\
& =360042 T_{7(4)+1}+75911 T_{7(3)+1}+5056 T_{7(2)+1} \\
& =25638893 T_{7(3)+1}+5405686 T_{7(2)+1}+360042 T_{7(1)+1} \\
& =1825767089 T_{7(2)+1}+384943437 T_{7+1}+25638893 T_{1} \\
& =5,742,568,741,225
\end{aligned}
$$

by plugging $T_{7(2)+1}=3136, T_{7+1}=44$ and $T_{1}=1$.

We note that, unlike the Fibonacci case in Theorem 2.2, the coefficients $\left(\mu_{1}, \mu_{2}, \mu_{3}\right)$ for tribonacci numbers in Theorem 3.1 and 3.2 depend on $k$. Now taking modular by tribonacci number $T_{k}$, the next corollary follows immediately. 
Corollary 3.6. Let $n=k t+r(1 \leq r \leq k)$. For $4 \leq k \leq 10$,

$$
T_{k t+r} \equiv \nu_{1} T_{k(t-1)+r}+\nu_{2} T_{k(t-2)+r}+\nu_{3} T_{k(t-3)+r} \quad\left(\bmod T_{k}\right)
$$

where the coefficients $\left(\nu_{1}, \nu_{2}, \nu_{3}\right)$ are

\begin{tabular}{l|l||l|l||l|l||l|l}
\hline$k$ & $\left(\nu_{1}, \nu_{2}, \nu_{3}\right)$ & $k$ & $\left(\nu_{1}, \nu_{2}, \nu_{3}\right)$ & $k$ & $\left(\nu_{1}, \nu_{2}, \nu_{3}\right)$ & $k$ & $\left(\nu_{1}, \nu_{2}, \nu_{3}\right)$ \\
\hline 4 & $(-1,1,1)$ & 5 & $(0,1,1)$ & 6 & $(0,2,1)$ & 7 & $(-1,15,1)$ \\
8 & $(-1,-1,1)$ & 9 & $(-2,-23,1)$ & 10 & $(-4,41,1)$ & & \\
\hline
\end{tabular}

EXAmple 3.7. For $T_{50}$, take $k=5$ and by $\bmod T_{5}=7$ for instance, $T_{50}=T_{5 \cdot 9+5} \equiv T_{5 \cdot 7+5}+T_{5 \cdot 6+5} \equiv\left(T_{5 \cdot 5+5}+T_{5 \cdot 4+5}\right)+T_{5 \cdot 6+5}$

$\equiv T_{5 \cdot 6+5}+T_{5 \cdot 5+5}+T_{5 \cdot 4+5} \equiv\left(T_{5 \cdot 4+5}+T_{5 \cdot 3+5}\right)+T_{5 \cdot 5+5}+T_{5 \cdot 4+5}$

$\equiv T_{5 \cdot 5+5}+2 T_{5 \cdot 4+5}+T_{5 \cdot 3+5} \equiv 2 T_{5 \cdot 4+5}+2 T_{5 \cdot 3+5}+T_{5 \cdot 2+5}$

$\equiv 2 T_{5 \cdot 3+5}+3 T_{5 \cdot 2+5}+2 T_{5+5} \equiv 3 T_{5 \cdot 2+5}+4 T_{5+5}+2 T_{5} \equiv 1$.

On the other hand, by taking different $k=10$, we have

$T_{50}=T_{10 \cdot 4+10} \equiv-4 T_{10 \cdot 3+10}+41 T_{10 \cdot 2+10}+T_{10 \cdot 1+10}$

$\equiv(56) T_{10+10}+98 T_{10}+57 T_{0} \equiv 56 \cdot 5 \equiv 131\left(\bmod T_{10}=149\right)$.

Corollary 3.5 yields $k$ columns modular tribonacci tables, for instance

\begin{tabular}{rrrr|rrrrr|rrrrrr}
\hline \multicolumn{1}{c|}{$\bmod \left(T_{4}=4\right)$} & \multicolumn{7}{|c|}{$\bmod \left(T_{5}=7\right)$} & \multicolumn{7}{|c}{$\bmod \left(T_{6}=13\right)$} \\
\hline \hline 1 & 1 & 2 & 0 & 1 & 1 & 2 & 4 & 0 & 1 & 1 & 2 & 4 & 7 & 0 \\
3 & 1 & 0 & 0 & 6 & 3 & 2 & 4 & 2 & 11 & 5 & 3 & 6 & 1 & 10 \\
1 & 1 & 2 & 0 & 1 & 0 & 3 & 4 & 0 & 4 & 2 & 3 & 9 & 1 & 0 \\
3 & 1 & 0 & 0 & 0 & 4 & 4 & 1 & 2 & 10 & 11 & 8 & 3 & 9 & 7 \\
1 & 1 & 2 & 0 & 0 & 3 & 5 & 1 & 2 & 6 & 9 & 9 & 11 & 3 & 10 \\
3 & 1 & 0 & $\cdots$ & 1 & 4 & 0 & 5 & $\cdots$ & 11 & 11 & 6 & 2 & 6 & $\cdots$ \\
\hline
\end{tabular}

THEOREM 3.8 .

(1) In the 4 columns modular tribonacci table

(i) $T_{4 t+r}+T_{4(t-1)+r} \equiv T_{4(t-2)+r}+T_{4(t-3)+r}\left(\bmod T_{4}=4\right)$.

(ii) $T_{4 t+4} \equiv 0$ and $T_{4 t+2} \equiv 1$ for every $t$

(iii) $T_{4 t+1} \equiv 1$ and $T_{4 t+3} \equiv 2$ if $t$ is even

(iv) $T_{4 t+1} \equiv 3$ and $T_{4 t+3} \equiv 0$ if $t$ is odd

(v) $(t)$ th row is congruent to $(t \pm 2)$ th row, i.e., $T_{k(t+2)+r} \equiv T_{k t+r}$.

(2) In the 5 columns modular tribonacci table

(i) $T_{5 t+r} \equiv T_{5(t-2)+r}+T_{5(t-3)+r}\left(\bmod T_{5}=7\right)$

(ii) $(t)$ th row is congruent to the sum of $(t-2)$ th and $(t-3)$ th rows.

Proof. In the 4 columns tribonacci table, Corollary 3.5 yields (i) that $T_{4 t+r} \equiv-T_{4(t-1)+r}+T_{4(t-2)+r}+T_{4(t-3)+r} \quad\left(\bmod T_{4}=4\right)$. 
We will only show (iv), and the rest can be proved similarly. Clearly $T_{4 t+1} \equiv 3$ if $t=1,3$. Assume $t$ is odd and $T_{4 i+1} \equiv 3\left(\bmod T_{4}\right)$ for all odd $i \leq t$. Then

$$
\begin{aligned}
T_{4(t+2)+1} & \equiv-T_{4(t+1)+r}+T_{4 t+r}+T_{4(t-1)+r} \\
& \equiv-\left(-T_{4 t+r}+T_{4(t-1)+r}+T_{4(t-2)+r}\right)+T_{4 t+r}+T_{4(t-1)+r} \\
& \equiv 2 \cdot 3-T_{4(t-2)+r} \equiv 2-\left(-T_{4(t-3)+r}+3+T_{4(t-5)+r}\right) \\
& \equiv-1+T_{4(t-3)+r}-T_{4(t-5)+r} \equiv 3 .
\end{aligned}
$$

We remark that in [5], the $4 n$ subscripted tribonacci numbers was proved that

$$
T_{4(n+1)}=11 T_{4 n}+5 T_{4(n-1)}+T_{4(n-2)}
$$

by mathematical induction. This is the case for $k=4$ in Theorem 3.1. In this sense Theorem 3.1 and 3.2 dealt with the $k n$ subscript tribonacci numbers for $4 \leq k \leq 10$. The identity $\sum_{t=0}^{n} T_{4 t}=\left(T_{4 n+4}+6 T_{4 n}+T_{4 n-4}-\right.$ $\left.T_{4}\right) / T_{4}^{2}$ was proved in [5] using matrix calculations. But Theorem 3.1 shows the identity easily.

Corollary 3.9. $T_{4}^{2} \sum_{t=0}^{n} T_{4 t}=T_{4 n+4}+6 T_{4 n}+T_{4 n-4}-T_{4}$.

Proof. Since $T_{4(3)+4}=\left(3 T_{4}-1\right) T_{4(2)+4}+\left(T_{4}+1\right) T_{4+4}+T_{4}$ by Theorem 3.1, $T_{4}^{2} \sum_{t=0}^{i} T_{4 t}=T_{4 i+4}+6 T_{4 i}+T_{4 i-4}-T_{4}$ is true if $i=3$. By induction we assume the equality holds for all $1 \leq i \leq n$. Then since $T_{4}=4$, it follows that

$$
\begin{aligned}
& T_{4(n+1)+4}+6 T_{4(n+1)}+T_{4(n+1)-4}-T_{4} \\
& =\left(3 T_{4}-1\right) T_{4 n+4}+\left(T_{4}+1\right) T_{4(n-1)+4}+T_{4(n-2)+4}+6 T_{4(n+1)}+T_{4(n+1)-4}-T_{4} \\
& =T_{4}^{2} T_{4 n+4}-\left(T_{4}+1\right) T_{4 n+4}+\left(T_{4}+1\right) T_{4(n-1)+4}+T_{4(n-2)+4} \\
& \quad+6 T_{4(n+1)}+T_{4(n+1)-4}-T_{4} \\
& =T_{4}^{2} T_{4 n+4}+T_{4(n+1)}+\left(T_{4}+2\right) T_{4 n}+T_{4 n-4}-T_{4} \\
& =T_{4}^{2} T_{4 n+4}+T_{4 n+4}+6 T_{4 n}+T_{4 n-4}-T_{4} \\
& =T_{4}^{2} T_{4 n+4}+T_{4}^{2} \sum_{t=0}^{n} T_{4 t}=T_{4}^{2} \sum_{t=0}^{n+1} T_{4 t} .
\end{aligned}
$$

\section{Matrix for modular Fibonacci sequence}

It is sometimes convenient to consider the $k$ columns Fibonacci table as the $k$ columns Fibonacci matrix. Then $F_{k t+r}$ can be regarded as the $(t+1)$ th row and $(r)$ th column entry $e_{(t+1, r)}$, so Theorem 2.2 implies that

$$
F_{k t+r}=e_{(t+1, r)}=\left(2 e_{(1, k)}+e_{(1, k-3)}\right) e_{(t, r)}+(-1)^{k-1} e_{(t-1, r)} .
$$


Hence $F_{k t+r}$ is a linear sum of three entries $e_{(1, k)}, e_{(1, k-3)}$ and $e_{(1, r)}$ in the 1st row, and $e_{(2, r)}$ in the 2nd row of $k$ columns Fibonacci matrix. Moreover $F_{k t+r}$ is expressed by two previous entries $e_{(t, r)}$ and $e_{(t-1, r)}$ in the same $(r)$ th column.

Theorem 4.1. Any Fibonacci number $F_{n}=F_{k t+r}$ is

$$
F_{k t+r} \equiv X M^{t-2}\left[\begin{array}{l}
e_{(1, r)} \\
e_{(2, r)}
\end{array}\right] \quad\left(\bmod F_{k}\right)
$$

where $X=\left[(-1)^{k-1} e_{(1, k-3)}\right]$ and $M=\left[\begin{array}{cc}0 & 1 \\ (-1)^{k-1} & e_{(1, k-3)}\end{array}\right]$. Moreover if let $a$ and $b$ be roots of $x^{2}-e_{(1, k-3)} x+(-1)^{k}=0$ then

$$
F_{k t+r} \equiv \frac{1}{a-b} X\left[\begin{array}{cc}
(-1)^{k-1}\left(a^{t-3}-b^{t-3}\right) & a^{t-2}-b^{t-2} \\
(-1)^{k-1}\left(a^{t-2}-b^{t-2}\right) & a^{t-1}-b^{t-1}
\end{array}\right]\left[\begin{array}{l}
e_{(1, r)} \\
e_{(2, r)}
\end{array}\right]
$$

Proof. In the $k$ columns Fibonacci matrix, by $\bmod F_{k}=e_{(1, k)}$,

$$
\begin{aligned}
& F_{k t+r} \\
&=e_{(t+1, r)} \\
& \equiv e_{(1, k-3)} e_{(t, r)}+(-1)^{k-1} e_{(t-1, r)} \\
& \equiv e_{(1, k-3)}\left(e_{(1, k-3)} e_{(t-1, r)}+(-1)^{k-1} e_{(t-2, r)}\right)+(-1)^{k-1} e_{(t-1, r)} \\
& \equiv {\left[e_{(1, k-3)}^{2}+(-1)^{k-1}\right] e_{(t-1, r)}+(-1)^{k-1} e_{(1, k-3)} e_{(t-2, r)} } \\
& \equiv {\left[e_{(1, k-3)}^{3}+2(-1)^{k-1} e_{(1, k-3)}\right] e_{(t-2, r)}+(-1)^{k-1}\left[e_{(1, k-3)}^{2}\right.} \\
&\left.+(-1)^{k-1}\right] e_{(t-3, r)} \\
& \equiv {\left[e_{(1, k-3)}^{4}+3(-1)^{k-1} e_{(1, k-3)}^{2}+(-1)^{2(k-1)}\right] e_{(t-3, r)} } \\
& \quad+(-1)^{k-1}\left[e_{(1, k-3)}^{3}+2(-1)^{k-1} e_{(1, k-3)}\right] e_{(t-4, r)}
\end{aligned}
$$

Continuing this process, $F_{k t+r}$ is expressed by means of matrices that

$$
\begin{aligned}
F_{k t+r} & \equiv\left[(-1)^{k-1} e_{(1, k-3)}\right]\left[\begin{array}{c}
e_{(t-1, r)} \\
e_{(t, r)}
\end{array}\right] \equiv X M\left[\begin{array}{l}
e_{(t-2, r)} \\
e_{(t-1, r)}
\end{array}\right] \\
& \equiv X M^{2}\left[\begin{array}{l}
e_{(t-3, r)} \\
e_{(t-2, r)}
\end{array}\right] \equiv X M^{3}\left[\begin{array}{l}
e_{(t-4, r)} \\
e_{(t-3, r)}
\end{array}\right] \equiv \cdots \\
& \equiv X M^{u}\left[\begin{array}{c}
e_{(t-u-1, r)} \\
e_{(t-u, r)}
\end{array}\right](\text { for } u \leq t-2) \equiv X M^{t-2}\left[\begin{array}{l}
e_{(1, r)} \\
e_{(2, r)}
\end{array}\right]
\end{aligned}
$$


where $M=\left[\begin{array}{cc}0 & 1 \\ (-1)^{k-1} & e_{(1, k-3)}\end{array}\right]$. Observe that $M=P D P^{-1}$ with $P=\left[\begin{array}{ll}1 & 1 \\ a & b\end{array}\right], D=\left[\begin{array}{ll}a & 0 \\ 0 & b\end{array}\right]$, with roots $a, b$ of $x^{2}-e_{(1, k-3)} x+(-1)^{k}=$ 0 . Thus $a+b=e_{(1, k-3)}$ and $a b=(-1)^{k}$, so

$$
M^{u}=P D^{u} P^{-1}=\frac{1}{a-b}\left[\begin{array}{cc}
-a b\left(a^{u-1}-b^{u-1}\right) & a^{u}-b^{u} \\
-a b\left(a^{u}-b^{u}\right) & a^{u+1}-b^{u+1}
\end{array}\right]
$$

and it proves the Theorem that

$$
F_{k t+r} \equiv \frac{1}{a-b} X\left[\begin{array}{cc}
(-1)^{k-1}\left(a^{t-3}-b^{t-3}\right) & a^{t-2}-b^{t-2} \\
(-1)^{k-1}\left(a^{t-2}-b^{t-2}\right) & a^{t-1}-b^{t-1}
\end{array}\right]\left[\begin{array}{l}
e_{(1, r)} \\
e_{(2, r)}
\end{array}\right] .
$$

Thus any $F_{k t+r}$ is obtained by $e_{(1, k-3)}, e_{(1, r)}, e_{(1, k)}$ and $e_{(2, r)}$, where the first three are in the 1st row and the last one is in the 2nd row in the $k$ columns Fibonacci matrix.

ExAmple 4.2. For $F_{99}$, consider $k=7$ for instance. Write $a$ and $b$ be roots of $x^{2}-e_{(1,4)} x-1=x^{2}-3 x-1=0$. Due to Theorem 4.1,

$$
\begin{aligned}
F_{99} & =F_{7(14)+1} \equiv \frac{1}{a-b}\left[\begin{array}{ll}
1 & e_{(1,4)}
\end{array}\right]\left[\begin{array}{cc}
0 & 1 \\
1 & e_{(1,4)}
\end{array}\right]^{12}\left[\begin{array}{l}
e_{(1,1)} \\
e_{(2,1)}
\end{array}\right] \\
& =\frac{1}{a-b}\left[\begin{array}{ll}
1 & 3
\end{array}\right]\left[\begin{array}{cc}
a^{11}-b^{11} & a^{12}-b^{-12} \\
a^{12}-b^{12} & a^{13}-b^{13}
\end{array}\right]\left[\begin{array}{l}
1 \\
8
\end{array}\right] .
\end{aligned}
$$

But since $a^{2}=3 a+1, a^{3}=3(3 a+1)+a=10 a+3$, we have $a^{11}=2 a+2$, $a^{12}=8 a+2$ and $a^{13}=8$. Hence

$a^{11}-b^{11} \equiv 2(a-b), \quad a^{12}-b^{12} \equiv 8(a-b), \quad a^{13}-b^{13} \equiv 0\left(\bmod F_{7}=13\right)$,

and so $F_{99}$ is congruent to

$$
\frac{1}{a-b}\left[\begin{array}{ll}
1 & 3
\end{array}\right]\left[\begin{array}{cc}
2(a-b) & 8(a-b) \\
8(a-b) & 0
\end{array}\right]\left[\begin{array}{l}
1 \\
8
\end{array}\right]=\left[\begin{array}{ll}
1 & 3
\end{array}\right]\left[\begin{array}{ll}
2 & 8 \\
8 & 0
\end{array}\right]\left[\begin{array}{l}
1 \\
8
\end{array}\right] \equiv 12 .
$$

In fact, $F_{99}=218,922,995,834,555,169,026 \equiv 12(\bmod 13)$.

The smallest integer $h>0$ satisfying $F_{h} \equiv 0$ and $F_{h+1} \equiv 1(\bmod n)$ is called the period of Fibonacci sequence by $\bmod n$. We write $h=$ $\operatorname{per}_{F}(n)$. Investigating the period of Fibonacci have been studied since Wall [7], so the period is usually called the Wall number by many researchers ([1]). A theorem about the period by mod Fibonacci numbers is as follows. 
TheOREM 4.3. $\operatorname{per}_{F}\left(F_{k}\right)=\left\{\begin{array}{ll}2 k & \text { if } k: \text { even } \\ 4 k & \text { if } k: \text { odd }\end{array}\right.$. In particular we have the table.

\begin{tabular}{l|l|l||r|l|l}
\hline$k$ & $F_{k}$ & $\operatorname{per}_{F}\left(F_{k}\right)$ & $k$ & $F_{k}$ & $\operatorname{per}_{F}\left(F_{k}\right)$ \\
\hline \hline 4 & 3 & $\operatorname{per}_{F}(3)=8=2 \cdot 4$ & 5 & 5 & $\operatorname{per}_{F}(5)=20=4 \cdot 5$ \\
\hline 6 & 8 & $\operatorname{per}_{F}(8)=12=2 \cdot 6$ & 7 & 13 & $\operatorname{per}_{F}(13)=28=4 \cdot 7$ \\
\hline 8 & 21 & $\operatorname{per}_{F}(21)=16=2 \cdot 8$ & 9 & 34 & $\operatorname{per}_{F}(34)=36=4 \cdot 9$ \\
\hline 10 & 55 & $\operatorname{per}_{F}(55)=20=2 \cdot 10$ & 11 & 89 & $\operatorname{per}_{F}(89)=44=4 \cdot 11$ \\
\hline 12 & 144 & $\operatorname{per}_{F}(144)=24=2 \cdot 12$ & 13 & 233 & $\operatorname{per}_{F}(233)=52=4 \cdot 13$ \\
\hline
\end{tabular}

The proof is due to Theorem 2.3 and Corollary 2.4. And Theorem 4.2 shows that period $\operatorname{per}_{F}\left(F_{k}\right)$ depends on only $k$ not on $F_{k}$, and is relatively short period comparing to the other $\operatorname{per}_{F}(n)$. For example,

$$
\operatorname{per}_{F}(987)=\operatorname{per}_{F}\left(F_{16}\right)=32, \operatorname{per}_{F}(1597)=\operatorname{per}_{F}\left(F_{17}\right)=68,
$$

however $\operatorname{per}_{F}(n)$ for $970 \leq n \leq 985$ is equal to

$2940,970,648,368,2928,1400,120,652,984$,

$220,1680,216,1470,1968,120,1980$

which show very long periods.

\section{References}

[1] A. Andreassian, Fibonacci sequence moduol M, Fibonacci Quarterly 12 (1974), no. $1,51-64$

[2] H. W. Austin, Columns of Fibonacci or Lucas Numbers, Mathematical Spectrum 37 (2005), 67-72.

[3] A. Ehrlich, On the periods of the Fibonacci sequence modulo M, Fibonacci Quarterly 27 (1989), no. 1, 11-13.

[4] J. H. Halton, On the divisibility properties of Fibonacci Number, Fibonacci Quarterly 4 (1966), no. 3, 217-240.

[5] E. Kilic, Tribonacci sequences with certain indices and their sums, Ars. Combinatorics 86 (2008), 13-22.

[6] A. Vince, The Fibonacci sequence modulo N, Fibonacci Quarterly 16 (1978), no. 4, 403-407.

[7] D. D. Wall, Fibonacci series modulo m, Amer. Math. Monthly 67 (1960), 525532 .

Department of Mathematics

HanNam University

Daejon 306-791, Republic of Korea

E-mail: emc@hnu.kr 